\title{
Ganoderma Lucidum Polysaccharide Peptide Alleviates Hepatoteatosis via Modulating Bile Acid Metabolism Dependent on FXR-SHP/FGF
}

\author{
Dandan Zhonga Zhengwei Xie ${ }^{a} \quad$ Boyue Huang ${ }^{a}$ Shuai Zhua Guoqian Wang ${ }^{a}$ \\ Hong Zhou ${ }^{a}$ Shuqian Lin ${ }^{b, c}$ Zhibin Lin ${ }^{a, b} \quad$ Baoxue Yang ${ }^{a, d}$ \\ aState Key Laboratory of Natural and Biomimetic Drugs, Department of Pharmacology, School of Basic

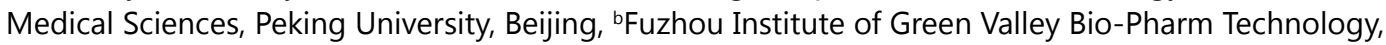 \\ Fuzhou, cJUNCAO Technology Research Institute, Fujian Agriculture and Forestry University, Fuzhou, \\ dKey Laboratory of Molecular Cardiovascular Sciences, Ministry of Education, Beijing, China
}

\section{Key Words}

Ganoderma lucidum polysaccharide peptide - NAFLD • Insulin resistance $\cdot$ hepatosteatosis • Metabolomics $•$ Bile acid synthesis $•$ Nuclear receptors $•$ Fatty acid synthesis

\begin{abstract}
Background/Aims: Non-alcoholic fatty liver disease (NAFLD) encompasses a series of pathologic changes ranging from steatosis to steatohepatitis, which may progress to cirrhosis and hepatocellular carcinoma. The purpose of this study was to determine whether ganoderma lucidum polysaccharide peptide (GLPP) has therapeutic effect on NAFLD. Methods: Ob/ $o b$ mouse model and ApoC3 transgenic mouse model were used for exploring the effect of GLPP on NAFLD. Key metabolic pathways and enzymes were identified by metabolomics combining with KEGG and PIUmet analyses and key enzymes were detected by Western blot. Hepatosteatosis models of HepG2 cells and primary hepatocytes were used to further confirm the therapeutic effect of GLPP on NAFLD. Results: GLPP administrated for a month alleviated hepatosteatosis, dyslipidemia, liver dysfunction and liver insulin resistance. Pathways of glycerophospholipid metabolism, fatty acid metabolism and primary bile acid biosynthesis were involved in the therapeutic effect of GLPP on NAFLD. Detection of key enzymes revealed that GLPP reversed low expression of CYP7A1, CYP8B1, FXR, SHP and high expression of FGFR4 in $o b / o b$ mice and ApoC3 mice. Besides, GLPP inhibited fatty acid synthesis by reducing the expression of SREBP1C, FAS and ACC via a FXR-SHP dependent mechanism. Additionally, GLPP reduced the accumulation of lipid droplets and the content of TG in HepG2 cells and primary hepatocytes induced by oleic acid and palmitic acid. Conclusion: GLPP significantly improves
\end{abstract}




\section{Cellular Physiology Cell Physiol Biochem 2018;49:1204-1220

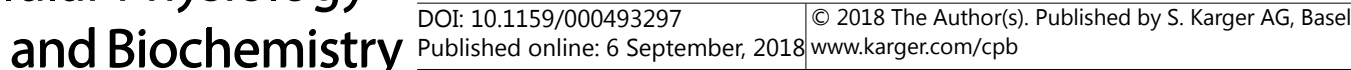 \\ Zhong et al.: GLPP Treats Non-Alcohol Fatty Liver Disease}

NAFLD via regulating bile acid synthesis dependent on FXR-SHP/FGF pathway, which finally inhibits fatty acid synthesis, indicating that GLPP might be developed as a therapeutic drug for NAFLD.

(C) 2018 The Author(s)

Published by S. Karger AG, Basel

\section{Introduction}

Non-alcoholic fatty liver disease (NAFLD) refers to hepatic steatosis accounting for more than $5 \% \sim 10 \%$ of the total weight of the liver or macrosteatosis of the same extent, which is not caused by excessive consumption of alcohol or other clear hepatotoxic factors [1]. NAFLD is a clinico-pathologically defined entity most commonly associated with obesity, insulin resistance (IR), diabetes, hypertension, dyslipidemia, atherosclerosis, systemic inflammation and other metabolic syndromes. NAFLD affects around $30 \%$ of the general population in developed countries and rises up to $70 \%$ in patients with type 2 diabetes mellitus (T2DM) or $90 \%$ in morbidly obese patients [2,3]. NAFLD encompasses a series of pathologic changes ranging from steatosis to steatohepatitis (NASH), which may progress to cirrhosis and hepatocellular carcinoma.

Currently there have been various therapies on NAFLD, such as changing diet, doing exercise to lose weight and using drugs like lipid-lowering drugs (statins), hypoglycemic drugs (thiazolidinedione and metformin) as well as antioxidants (vitamin E and acetylcysteine). However, all of these therapies are hampered by some limitations and side effects [4]. So far, no single therapy is able to halt or reverse NAFLD or NASH. It is necessary to discover novel drugs and effective therapies for NAFLD.

Ganoderma lucidum (also known as lingzhi or reishi) is a traditional Chinese medicine that has been consumed for its broad medicinal properties in Asia for thousands of years [5]. Ganoderma lucidum has been widely used for prevention and treatment of various medical diseases including cancer [6, 7], liver damage induced by hepatotoxic drugs [8, 9], kidney injury [10], specifically for metabolic diseases such as diabetes [11], obesity [12], and other cardiovascular syndromes [13]. Ganoderma lucidum polysaccharide peptide (GLPP), a group of extract from Ganoderma lucidum (Leyss ex Fr.) Karst with a molecular weight of approximately $5 \times 10^{5}$, which ratio of polysaccharide to peptide is approximately $95 \%$ / $5 \%$ [14]. GLPP is one of the major pharmacological constituent of Ganoderma lucidum and has diverse bioactivities [15]. Our previous study revealed that GLPP prevented renal ischemia reperfusion injury via counteracting oxidative stress [10]. Besides, a randomized, doubleblind placebo-controlled crossover study indicated that polysaccharide peptides enriched Ganoderma lucidum possessed antioxidation and hepatoprotection in healthy volunteers [16]. However, whether GLPP can protect against NAFLD and its related mechanism remain unknown.

In the present study, we used $o b / o b$ mice and apolipoprotein CIII (ApoC3) transgenic mice as in vivo experimental models to evaluate the effect of GLPP on NAFLD, and elucidated the mechanisms using metabolomics combined with KEGG and PIUmet analysis. HepG2 cells and primary hepatocytes induced by oleic acid and palmitic acid as in vitro models were used to convince the evidence of GLPP on hepatic steatosis. Our results showed that GLPP had therapeutic effect on NAFLD via changing the classic pathway of bile acids (BAs) synthesis regulated by an FXR-SHP/FGF dependent mechanism and inhibiting the downstream pathway of fatty acid synthesis. Experimental results suggest that GLPP is a candidate drug for NAFLD treatment.

\section{Materials and Methods}

Reagents and antibodies

GLPP is a hazel-colored, water-soluble powder, kindly provided by Fuzhou Institute of Green Valley Bio-Pharm Technology. The average molecular weight of GLPP is approximately $520 \mathrm{kDa}$, as determined 


\section{Cellular Physiology Cell Physiol Biochem 2018;49:1204-1220

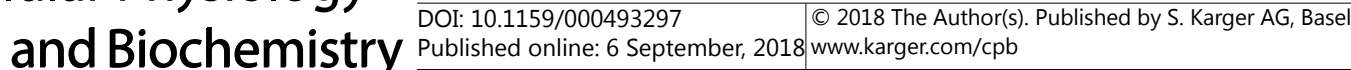 \\ Zhong et al.: GLPP Treats Non-Alcohol Fatty Liver Disease}

by high-performance steric exclusion chromatography analysis. In the experiments, GLPP was dissolved in physiological saline for animal treatment and in PBS for cell incubation. Oleic acid (01008, Sigma, St. Louis, MO), palmitic acid (P5585, Sigma, St. Louis, MO) and oil red O (00625, Sigma, St. Louis, MO) were purchased from Sigma. Antibodies against the following proteins were purchased from Cell Signaling Technology: FAS (3180, 1:1000 dilution), IRS2 (4502, 1:1000 dilution), p-GSK3 $\beta$ (9336s, 1:1000 dilution), GSK3 $\beta$ (9315s, 1:1000 dilution); Antibodies against the following proteins were purchased from Abcam: p-IRS2 (ab3690, 1:1000 dilution), p-AKT2 (ab38513, 1:1000 dilution); Antibodies against the following proteins were purchased from Novusbio: SREBP-1c (NB600-582, 1:1000 dilution); Antibodies against the following proteins were purchased from Millipore: ACC (05-1098, 1:1000 dilution); Antibodies against the following proteins were purchased from ABclonal: AKT (A3145, 1:1000 dilution), CPT1A (A5307, 1:1000 dilution), SHP (A1836, 1:1000 dilution); Antibodies against the following proteins were purchased from Bioss: CYP7A1 (bs-2399R, 1:1000 dilution); Antibodies against the following proteins were purchased from Proteintech: CYP7B1 (24889-1-AP, 1:1000 dilution), LCAT (12243-1-AP, 1:1000 dilution); Antibodies against the following proteins were purchased from Huaxingbio: CYP8B1 (HX13801, 1:1000 dilution), LPGAT1 (HX19808, 1:1000 dilution), CLC (HX19901, 1:1000 dilution), LXR $\alpha$ (HX15716, 1:1000 dilution), FXR (HX19905, 1:1000 dilution), FGFR4 (HX14373, 1:1000 dilution); Antibodies against the following proteins were purchased from Wanlei bio: HNF4 $\alpha$ (WL01575, 1:5000 dilution); Antibodies against the following proteins were purchased from Easybio: $\beta$-tubulin (B1031, 1:5000 dilution).

\section{Animals}

Male $o b / o b$ mice on a C57BL6/J genetic background and C57BL6/J wild-type littermates at 8 weeks of age were purchased from the Animal Center of Peking University Health Science Center. ApoC3 transgenic mice on a C57BL6/J genetic background and C57BL6/J wild-type littermates at 12 weeks of age were kindly provided by Guoqing Liu. Mice were housed in a temperature-controlled environment $\left(23 \pm 2{ }^{\circ} \mathrm{C}\right)$ with a cycle of $12 \mathrm{~h}$ of light followed by $12 \mathrm{~h}$ of dark and free access to food and water. Mice were randomly distributed into four groups: (1) C57BL6/J wild-type controls administrated with saline; (2) C57BL6/J wildtype controls administrated with GLPP; (3) ob/ob mice or ApoC3 transgenic mice administrated with saline; and (4) $o b / o b$ mice or ApoC3 transgenic mice administrated with GLPP. Mice were given orally with GLPP at a dosage of $100 \mathrm{mg} / \mathrm{kg} /$ day or saline vehicle for four weeks until sacrifice. Body weight was determined weekly and animals were killed in a non-fasted state at the end of experiments. All animal experiments were conformed to the Guide for the Care and Use of Laboratory Animals published by the US National Institutes of Health (NIH Publication, eighth Edition, 2011) and was approved by the PUHSC Animal Experimentation Ethics Committee (Laboratory animal use license No. XYSK (JING) 2011-0039, Laboratory animal production license No. SCXK (JING) 2011-0012).

\section{Glucose tolerance test (GTT) and insulin tolerance test (ITT)}

For GTT, mice were fasted for $6 \mathrm{~h}$, and dextrose $(1.5 \mathrm{~g} / \mathrm{kg})$ was administered intraperitoneally. Blood glucose levels were measured from the tail before dextrose administration and 15, 30, 60, 90 and $120 \mathrm{~min}$ after administration. For ITT, mice were fasted for $6 \mathrm{~h}$, starting at $8 \mathrm{a}$ am. and lasting until $2 \mathrm{p}$.m. Recombinant human insulin (1 IU $/ \mathrm{kg}$ ) was administered intraperitoneally. Blood glucose levels were measured from the tail before insulin administration and 15, 30, 60, 90 and 120 min after the administration.

\section{Collection of serum and tissues samples}

At the end of experiment, the blood was collected via venae orbitaeta and centrifuged at $5000 \mathrm{rpm}$ for $10 \mathrm{~min}$ at $4{ }^{\circ} \mathrm{C}$. Serum was stored at $-80^{\circ} \mathrm{C}$ for metabolomics analysis and biochemistry assay. After euthanizing the mice, the liver, kidney, spleen, visceral fat and epididymal adipose tissue were collected for histopathological observation or stored at $-80^{\circ} \mathrm{C}$ having been flash-frozen until analysis.

\section{Biochemical assay}

We measured plasma glucose via assay kit (Shanghai Rongsheng Biotech Co., Ltd) according to the manufacturer's instructions. Plasma TG, TC, HDL-c, LDL-c, ALT and AST were measured by using automatic analyzer (AU5800 comes with ion test reagent, Beckman Coulter Commercial Enterprise CO., Ltd.). 


\section{Cellular Physiology Cell Physiol Biochem 2018;49:1204-1220

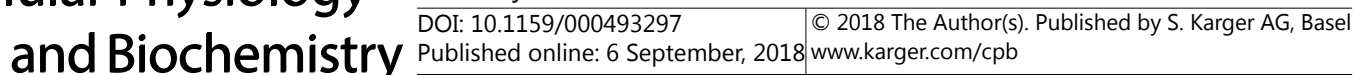 \\ Zhong et al.: GLPP Treats Non-Alcohol Fatty Liver Disease}

Analysis of hepatic lipid content

For hepatic lipid extraction, liver samples were weighed and homogenized. Four milliliters of chloroform/methanol $(2 / 1, \mathrm{v} / \mathrm{v})$ was added to the homogenization and vortexed for $30 \mathrm{~s}$. The resultant mixture was centrifuged at $836 \times \mathrm{g}$ for $30 \mathrm{~min}$. Then the supernatant was taken out to a new tube for further extraction of lipids using the same method as mentioned before. The lower layer of lipid extraction was taken to another new tube. After the organic solvents volatilized completely, the residual was dissolved in $500 \mu \mathrm{L} 3 \%$ Triton-X 100. Total TC and TG content in the resultant solution were determined according to the instruction of commercial kits (Applygen).

\section{Histological analysis}

Liver and fat tissues were fixed in $4 \%$ paraformaldehyde and embedded in paraffin and $5 \mu \mathrm{m}$ sections were cut and stained with $\mathrm{H} \& \mathrm{E}$ staining for histological analysis. Liver samples embedded in OCT compound (Tissue-Tek ${ }^{\circledR}$ OCT ${ }^{\mathrm{TM}}$ Compound, Sakura Finetek USA) were cut and stained with oil red 0 and counterstained with hematoxylin to visualize intracellular droplets. All digital images were obtained with a light microscope (Leica, Wetzlar, Hesse-Darmstadt, Germany).

\section{Oil red semi-quantitative analysis}

Lipid droplets were stained by red color in background of white and nucleus were colored in blue. Lipid content was quantified by the outgrow of red channel. After substrating minimum values of three color channels, the residue of red channel was treated as the amount of lipid content. Similarly, residue of blue channel was treated as amount of nucleus. All regions were analyzed equally. The sample of code is shown as followed.

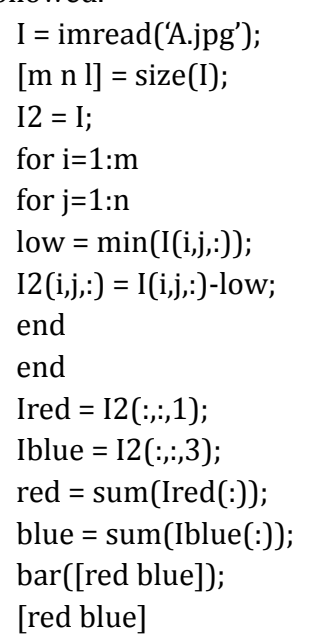

\section{Untargeted metabolomics analysis}

Serum obtained from all mice ( $\mathrm{n}=8$, each group) was analyzed by LC/MS/MS to identify metabolites using a library of over 1000 compounds. For the pretreatment of the serum samples, serum was thawed on ice and mix with 3 times volume of acetonitrile. The mixture was allowed to stand for $10 \mathrm{~min}$ after vortexing for $60 \mathrm{~s}$, and then the samples were centrifuged at $13000 \mathrm{rpm}$ for $10 \mathrm{~min}$ at $4{ }^{\circ} \mathrm{C}$. The supernatants were filtered through syringe filters $(0.22 \mu \mathrm{m}$, Jinteng) before UPLC-MS analysis. Metabolites profiling was performed by Metabolon (Durham, NC) using ultra high performance liquid chromatography and tandem mass spectrometry (UPLC-MS) optimized for the detection of positive or negative metabolites, and gas chromatography (GC)/MS. The UPLC-MS platform was based on a Waters ACQUITY UHPLC and ThermoFinnigan LTQ mass spectrometer, which consisted of an electrospray ionization source and linear ion-trap mass analyzer. Analysis was performed on a ThermoFinnigan Trace DSQ fast-scanning single-quadrupole mass spectrometer using electron impact ionization. Compounds were identified by automated comparison of chromatographic and mass spectra properties in samples to metabolomic library entries of purified standards. 


\section{Cellular Physiology Cell Physiol Biochem 2018;49:1204-1220 and Biochemistry $\begin{gathered}\text { DOI: 10.1159/000493297 } \\ \text { Published online: 6 September, } 2018 \text { www.karger.com/cpb }\end{gathered}$ \\ Zhong et al.: GLPP Treats Non-Alcohol Fatty Liver Disease}

Data processing and metabolite selection

The raw data were imported to Markerlynx software (Waters Corp, MA, USA.) for peak detection and alignment to obtain a peak list containing the retention time, $\mathrm{m} / \mathrm{z}$, and peak area of each sample. Significantly changed metabolites were calculated by computing ANOVA models and using Student's $t$ statistics (unpaired and unequal variance). A significance threshold of $p<0.05$ was applied. Adjustment of $\mathrm{p}$ values according to Benjamini and Hochberg was applied to correct for multiple testing. Metabolites were categorized based on their significance level in both mouse models or their specific change in either of the models compared with respective wild-type mice or GLPP-treated mice compared with saline-treated mice. Principal component analysis (PCA), partial least squares discriminant analysis (PLS-DA) and orthogonal partial least squares discriminant analysis (OPLS-DA) were used to identify the major metabolites. The variable importance in the projection (VIP) value of each variable in the model was calculated to indicate its contribution to the classification. VIP values $>1.0$ were considered significantly different based on their contribution to the variation and correlation within the data set. Odds ratios and $95 \%$ confidence intervals were calculated. Open database sources, including the KEGG compound database, lipidmaps database and human metabolomedatabase were used to identify metabolic pathways. PIUmet analysis developed by Pirhaji et al. [17] was then used to identify key enzymes behind up-regulated metabolites or down-regulated metabolites separately.

\section{Western blot analysis}

Tissues or cells were lysed in RIPA lysis buffer (Applygen, Beijing, China) containing $4 \%$ protease inhibitor cocktail (Roche, Indianapolis, IN, USA) and $1 \%$ protein phosphatase inhibitor (Applygen). The extract was homogenized using a Dounce homogenizer and centrifuged at 12, $000 \mathrm{rpm}$ for $20 \mathrm{~min}$ at 4 ?. Isolated total protein was measured by BCA assay (Pierce Biotechnology, Rockford, IL, USA) and separated by SDS-polyacrylamide gel electrophoresis. Proteins were then blotted to polyvinylidene difluoride membranes (Amersham Biosciences, Piscataway, NJ, USA). Blots were incubated with antibodies against indicated antibodies (See Reagents and antibodies) in $4^{\circ} \mathrm{C}$ for overnight. Afterwards, goat anti-rabbit IgG (Abcam) or goat anti-mouse IgG (Santa Cruz Biotechnology) was added for one hour in room temperature, and then the blots were developed with ECL Plus Kit (Amersham Biosciences). Relative protein expression was quantified by optical density using Quantity One.

\section{ELISA}

Supernatants of homogenized livers were prepared by using RIPA lysis buffer and centrifuged at 3000 rpm for 20 minutes. Levels of p-AKT2 and AKT in liver were measured by commercially available ELISA kits, according to the protocols of the manufacturer (mouse AKT ELISA kit, EXP210475 or mouse phospho-AKT2 ELISA kit, EXP210671). Three samples in each group were chosen and each sample was measured in three repeats (average values were used in our analysis).

\section{Cell lines and culture}

Human hepatoma cell line HepG2 cells were cultured at $37{ }^{\circ} \mathrm{C}$ with $5 \% \mathrm{CO}_{2}$ in humidified atmosphere. After planted for $24 \mathrm{~h}$, HepG2 cells were exposed to a mixture of $0.6 \mathrm{mM}$ oleic acid and palmitic acid (2:1) for $12 \mathrm{~h}$ to induce cell hepatosteatosis model. GLPP was added for $24 \mathrm{~h}, 48 \mathrm{~h}$ or $72 \mathrm{~h}$ to detect the therapeutic effect on NAFLD. Primary mouse hepatocyte culture: hepatocytes were isolated from C57 mice by nonrecirculating collagenase perfusion through the portal vein as previously described with little modification [18]. Briefly, mice were anesthetized and perfused with freshly prepared perfusion medium (Invitrogen, Carlsbad, CA, USA) via the portal vein and then followed by digestion buffer (collagenase IV, $0.1 \mathrm{mg} / \mathrm{ml}$, Worthington, NJ, USA). Then perfused livers were removed from the mice and dispersed with hepatocyte wash medium (Invitrogen, Carlsbad, CA, USA). The viable cells were counted by trypan blue exclusion assay and then seeded onto fibronectin-coated plates (Corning, Rochester, NY). Medium was changed after planted for $4 \mathrm{~h}$, and cell hepatosteatosis model was induced by co-incubation with a mixture of $0.3 \mathrm{mM}$ oleic acid and palmitic acid (2:1) for $12 \mathrm{~h}$. GLPP was added for another $24 \mathrm{~h}$ or $48 \mathrm{~h}$ to test the therapeutic effect on NAFLD. 


\section{Statistical analysis}

Statistical analyses were performed using GraphPad Prism software. All results are represented as the mean \pm SEM. Each experiment was performed at least 3 times. Data involving only two groups were analyzed using a twotailed Student $t$-test assuming unequal variances. When more than two experimental groups were compared, the data were analyzed using the Tukey-Kramer test with Prism 5.0 software to compare data between individual experimental groups. A p-value of $<0.05$ was considered to be statistically significant for all tests.

\section{Results}

\section{GLPP ameliorates} hepatosteatosis and liver dysfunction in $o b /$ ob mice

$\mathrm{Ob} / \mathrm{ob}$

mice

characterized with obesity, steatosis, IR and other metabolic syndromes are one of the most typical models for studying NAFLD. Compared to control mice, $o b / o b$ mice presented increased liver size, liver weight, liver triglyceride (TG) and total-cholesterol (TC) (Fig. 1 a-d). Besides, $o b / o b$ mice had significant dyslipidemia (Fig. 1 e-h). $H \& E$ staining showed that the liver of $o b / o b$ mice had apparent hepatosteatosis, including massive accumulation of large lipid droplets and ballooning degeneration of liver cells (Fig. 1 i). The oil red 0 staining indicated that more lipid droplets accumulated in the liver of $o b / o b$ mice (Fig. $1 \mathrm{j}, \mathrm{k}$ ). Blood alanine aminotransferase (ALT) and aspartate aminotransferase (AST) were significantly increased in $o b / o b$ mice compared to control mice (Fig. $1 \mathrm{l}, \mathrm{m}$ ). Data suggest that $o b / o b$ mice appear significant dyslipidemia, liver steatosis and liver dysfunction. After treatment of GLPP for 4 weeks, liver size and weight were reduced in GLPP-treated mice (Fig. 1 a,b). Liver TG and TC were significantly decreased and dyslipidemia was improved after GLPP treatment (Fig. $1 \mathrm{c}-\mathrm{h}$ ). $\mathrm{H} \& \mathrm{E}$ staining and oil red $\mathrm{O}$ staining showed that GLPP alleviated hepatosteatosis and lipid

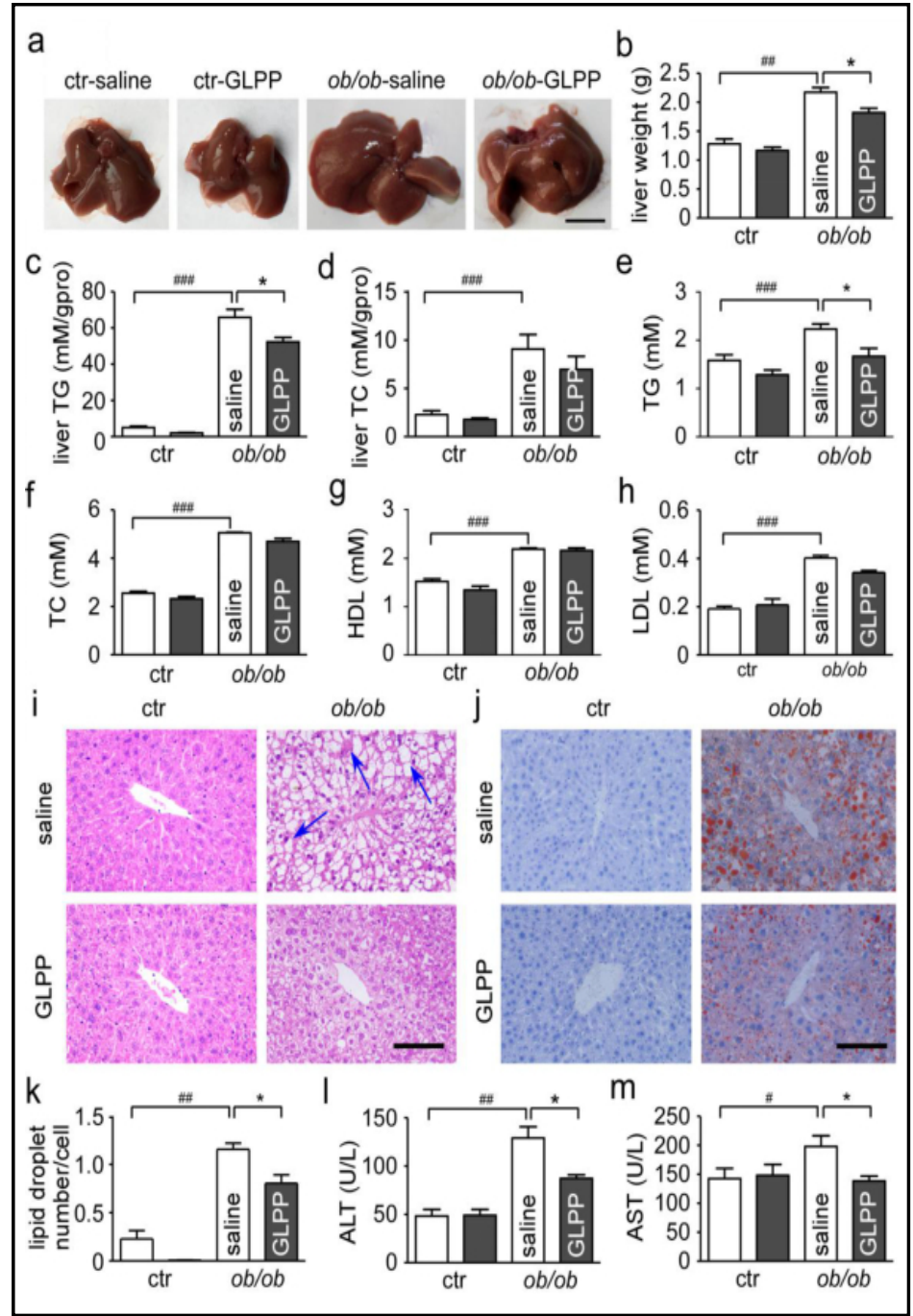

Fig. 1. GLPP alleviates hepatosteatosis and improves liver function in ob/ob mice. Eight-week-old ob/ob mice administered with either saline or GLPP for 4 weeks. (a) Representative image of liver gross appearance. (b) Liver weight $(n=8)$. (c) Total triglyceride level in the liver ( $\mathrm{n}=8)$. (d) Total cholesterol level in the liver $(\mathrm{n}=8)$. (e) Blood TG. (f) Blood TC. (g) Blood HDL. (h) Blood LDL. (i) H \& E staining of liver tissue section. Scale bar, $100 \mu \mathrm{m}$. (j) Oil red 0 staining of liver tissue section. Scale bar, $100 \mu \mathrm{m}$. (k) Quantification of lipid content in liver (n =3). (l) ALT level $(\mathrm{n}=8)$. (m) AST level $(\mathrm{n}=8)$. Quantitative data are presented as mean $\pm \mathrm{SEM}$. ${ }^{*} \mathrm{P}<0.05$, ${ }^{\#} \mathrm{P}<0.05$, ${ }^{\# \#} \mathrm{P}<0.01,{ }^{\# \# \#} \mathrm{P}<0.001$. 
deposition (Fig. 1 i-k). Also, we found that GLPP decreased both ALT and AST (Fig. 1 l, m), which suggests that GLPP not only ameliorates the liver steatosis but also improves liver function.

GLPP reduces visceral fat accumulation and improves liver insulin resistance in ob/ob mice Adipose tissue accumulation in the viscera is closely associated with NAFLD [19]. We further explored the effect of GLPP on lipid accumulation in peripheral tissues. As results showed, GLPP reduced the weight of mesentery fat (Fig. 2 a), but had no significant influence on weight of other organs and epididymal fat (data not show). H\&E staining showed that GLPP reduced the diameters of adipocytes of mesentery fat (Fig. 2 b,c). Additionally, considering that NAFLD is accompanied by IR, we performed glucose tolerance test (GTT) and insulin tolerance test (ITT). As results showed, glucose tolerance was significantly improved after GLPP treatment (Fig. 2 d, e), while no obvious difference of GLPP on insulin tolerance (Fig. 2 f, g). IR in whole body was complex and easily disturbed by a variety of factors, we then analyzed the effect of GLPP on the liver IR. Since AKT plays key roles in regulating IR, we conducted ELISA analysis

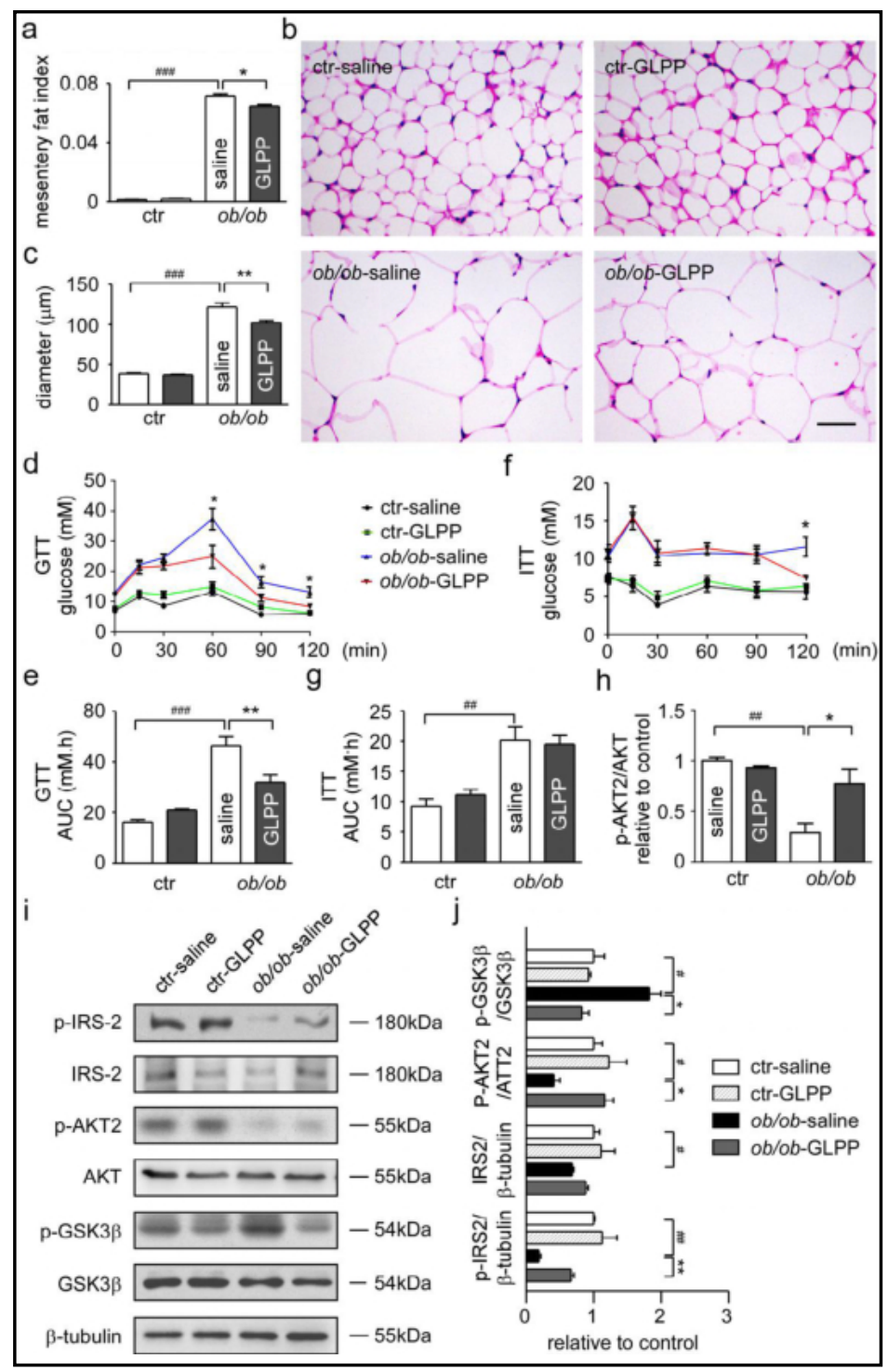

Fig. 2. GLPP alleviates the liver insulin resistance in ob/ob mice. (a) Ratio of mesentery fat weight to body weight $(n=8)$. (b) H \& E staining of mesentery fat. Scale bar, $100 \mu \mathrm{m}$. (c) Statistic analysis of the diameters of mesentery fat according to Image J. (n=3). (d) Intraperitoneal glucose tolerance test (GTT) $(n=6-8), *$ means ob/ob-saline versus ob/ob-GLPP. (e) Blood glucose area under the curve (AUC) ( $n=6-8$ ). (f) Intraperitoneal insulin tolerance test (ITT) ( $n=6-8), *$ means ob/ ob-saline versus ob/ob-GLPP. (g) Blood insulin area under the curve (AUC) (n = 8). (h) Relative level of p-AKT2/AKT measured by ELISA $(n=6)$. (i) Representative Western blotting showing the expression pattern of proteins involved in liver IR. (j) Quantification of signaling proteins shown in (i) $(\mathrm{n}=3)$. Quantitative data are presented as mean \pm SEM. ${ }^{*} \mathrm{P}<0.05,{ }^{* *} \mathrm{P}<0.01$. ${ }^{\#} \mathrm{P}<0.05,{ }^{\# \#} \mathrm{P}<0.01,{ }^{\# \#} \mathrm{P}<0.001$. and found that $\mathrm{p}-\mathrm{AKT} 2 / \mathrm{AKT}$ decreased in $o b / o b$ mice while GLPP reversed the change, which indicates that AKT is involved in liver IR (Fig. $2 \mathrm{~h}$ ). Previous studies reported that the impairment of insulin sensitivity is largely mediated by insulin signaling, in particular the IRS-AKT-GSK3 $\beta$ cascades [20]. We then tested expression of insulin receptor substrate 2 (IRS2), AKT2 and glycogen synthase kinase- 
$3 \beta($ GSK3 $\beta$ ) and found that IRS2, phosphorylation of IRS2 and AKT2 decreased while phosphorylation of GSK3 $\beta$ increased in the liver of $o b / o b$ mice, which suggested that $o b / o b$ had undergone serious liver IR. However, GLPP reversed the expression of those proteins and ameliorated the impaired insulin signaling pathway in the liver (Fig. 2 $\mathrm{i}, \mathrm{j}$ ).

GLPP improves liver steatosis and insulin resistance in ApoC3 transgenic mice

We then used ApoC3 transgenic mice, a model with genetic predisposition to hypertriglyceridemia and mild steatosis, to further convince the protection of GLPP on hepatic steatosis and its related metabolic disorders. In ApoC3 transgenic mice, GLPP ameliorated dyslipidemia and liver lipids accumulation (Fig. 3 a-f). H\&E staining showed significant hepatocyte ballooning in ApoC3 transgenic mice while restored nearly to normal after GLPP treatment (Fig. $3 \mathrm{~g})$. The oil red 0 staining showed that GLPP reduced lipid droplets deposition compared to ApoC3 transgenic mice (Fig. $3 \mathrm{~h}$, i). Moreover, we confirmed the results that GLPP could alleviate the abnormal liver function via decreasing the levels of ALT and AST in ApoC3 transgenic mice (Fig. $3 \mathrm{j}, \mathrm{k}$ ).

Moreover, we found p-AKT2/AKT2 decreased in ApoC3 mice, as measured by ELISA, which suggests that AKT signaling pathway might be inhibited in ApoC3 mice (Fig. 3 l). We then detected the expression of key proteins in IRS-

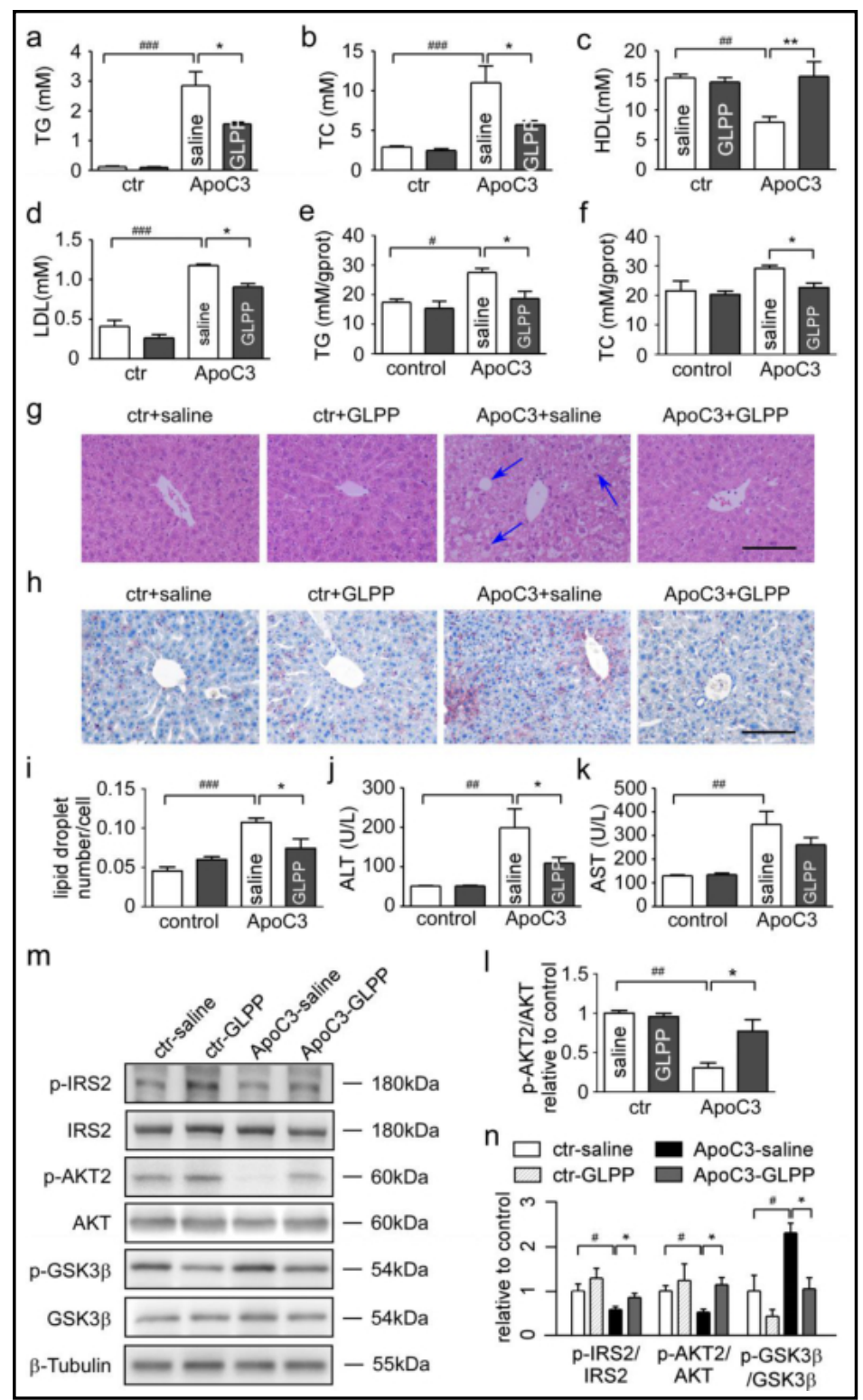

Fig. 3. GLPP ameliorates liver steatosis and improved liver insulin resistance in ApoC3 mice. ApoC3 mice and their littermate control mice were administered with either saline or GLPP for 4 weeks. (a) Blood TG. (b) Blood TC. (c) Blood HDL. (d) Blood LDL.(e) Total triglyceride levels in liver $(n=8)$. (f) Total cholesterol levels in liver $(n=8)$. (g) $\mathrm{H} \& \mathrm{E}$ staining of liver tissue section. Scale bar, $100 \mu \mathrm{m}$. (h) Oil red 0 staining of liver tissue section. Scale bar, $100 \mu \mathrm{m}$. (i) Quantification of lipid content in liver $(n=3)$. (j) ALT level $(n=8)$. (k) AST level $(n=$ 8) (l) Relative level of p-AKT2/AKT measured by ELISA $(n=6) .(\mathrm{m})$ Representative Western blotting showing the expression pattern of proteins involved in the liver insulin resistance. (n) Quantification of proteins as in $(\mathrm{m})(\mathrm{n}=3)$. Quantitative data are presented as mean \pm SEM. ${ }^{*} \mathrm{P}<0.05,{ }^{* *} \mathrm{P}<0.01$. ${ }^{\#} \mathrm{P}<0.05,{ }^{\# \#} \mathrm{P}<0.01,{ }^{\# \#} \mathrm{P}<0.001$. 
Fig. 4. GLPP influences the bile acid synthesis and fatty acid metabolism. (a) Pathways of metabolites charged positive enriched by KEGG analysis. (b) Pathways of metabolites charged negative enriched by KEGG analysis. 1: Glycerophos pholipid metabolism; 2: Linoleic acid metabolism; 3: Thiamine metabolism; 4: Sphingolipid metabolism; 5: Synthesis and degradation of ketone bodies; 6: Taurine and hypotaurine metabolism: 7: Fatty acid elongation in mitochondrial; 8: primary bile acid biosynthesis; 9: Fatty acid biosynthesis. (c) Key enzymes enriched by up-regulated metabolites according to PIUmet analysis (d) Key enzymes enriched by downregulated metabolites according to PIUmet analysis. (e) representative blotting of proteins or enzymes showed in (c) and (d) in ob/ob mice. (f) representative blotting of proteins or enzymes showed in (c) and (d) in ApoC3 mice. (g) Quantification of signaling proteins as in (e) $(n=$ 3). (h) Quantification of signaling proteins as in (f) $(\mathrm{n}=3)$. Quantitative data are presented as mean \pm SEM. $\quad * \mathrm{P}<0.05, \quad * * \mathrm{P}<0.01$, ${ }^{\#} \mathrm{P}<0.05$, ${ }^{\# \#} \mathrm{P}<0.01$.

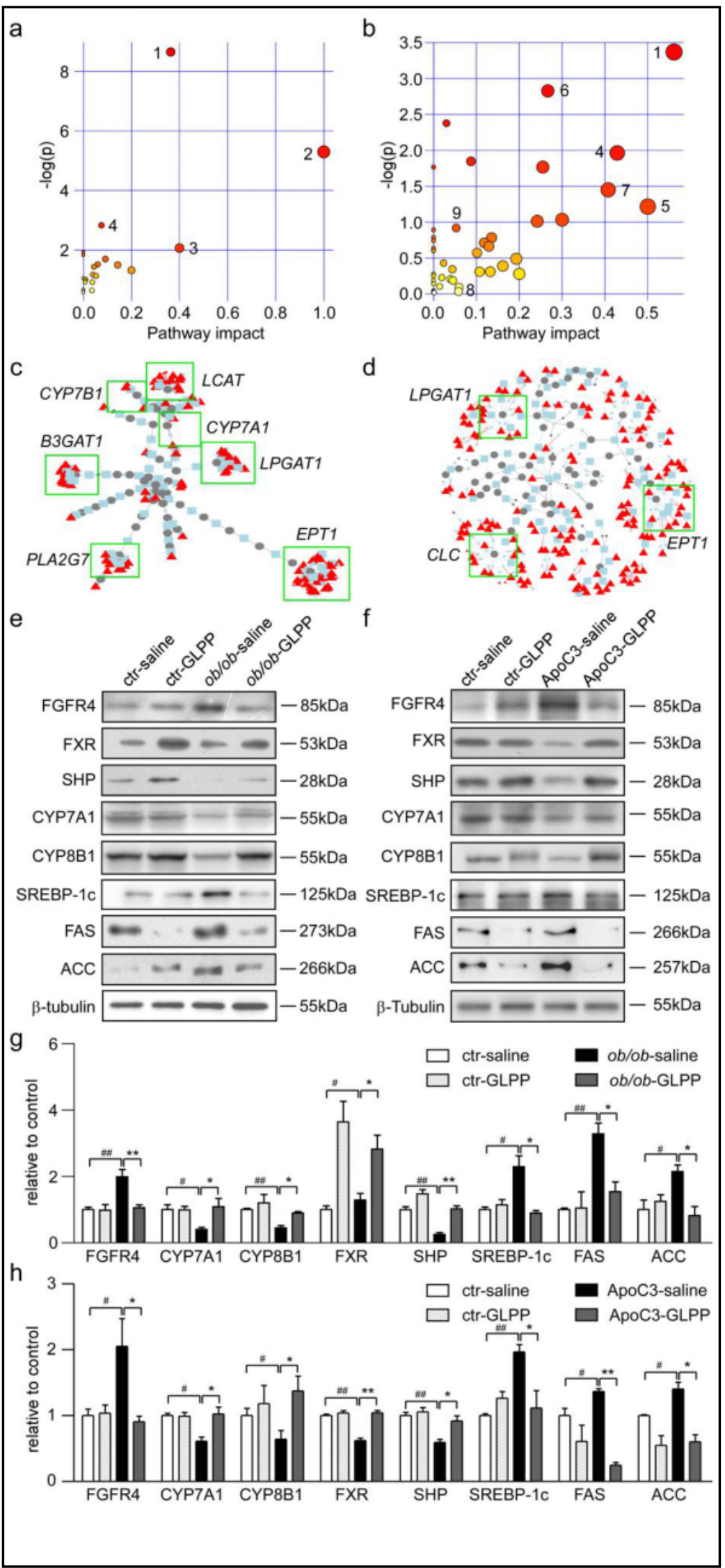


AKT-GSK3 $\beta$ signaling pathway. As results showed, the levels of p-IRS2 and p-AKT2 decreased while the level of p-GSK3 $\beta$ increased in ApoC3 mice. However, GLPP reversed the regulated levels (Fig. $3 \mathrm{~m}, \mathrm{n}$ ). The results suggest that GLPP may ameliorate the liver IR via modifying the impaired axis of IRS2-AKT2-GSK3 $\beta$.

\section{GLPP regulates bile acids metabolism and inhibits fatty acid synthesis}

Metabolite profiles obtained from serum of $o b / o b$ mice were tested to explore the mechanism behind the protective effect of GLPP against NAFLD. Biomarkers that significantly changed were recognized and were mapped on KEGG (http://www.kegg.jp/kegg/) to form a metabolic network, giving a holistic view of metabolites changes involved in the organism. They were mainly involved in glycerophospholipid metabolism, taurine and hypotaurine metabolism, fatty acid metabolism and primary bile acid biosynthesis (Fig. 4 a, b) which are closely associated with the development and progression of NAFLD. To identify enzymes behind the scene, we employed a network-based approach PIUMet developed by Pirhaji et al. [17] PIUmet encloses a sub-metabolic network defined by up-regulated metabolites (Fig. $4 \mathrm{c}$ ) or down-regulated metabolites (Fig. $4 \mathrm{~d}$ ). The possible pathways and key enzymes were determined according to the KEGG and PIUmet analysis. There was no significant difference in expression levels of 25-hydroxycholesterol 7-alpha-hydroxylase (CYP7B1), chloride channel (CLC), lecithin cholesterol acyltransferase (LCAT) and lysophosphatidylglycerol acyltransferase1 (LPGAT1) between each group (data not show). However, cholesterol 7-alpha-monooxygenase (CYP7A1) and 7-alpha-hydroxycholest-4-en-3-one 12-alphahydroxylase (CYP8B1) levels decreased in ob/ob mice and ApoC3 transgenic mice while GLPP increased their expression (Fig. 4 e-h), which indicates that the classic pathway of BAs synthesis was involved in the protection of GLPP against NAFLD. CYP7A1, the rate-limiting enzyme of BAs synthesis is regulated by many nuclear receptors and transcriptional factors. We then analyzed the expression of farnesoid $\mathrm{X}$ receptor (FXR), liver $\mathrm{X}$ receptor (LXR) and small heterodimer partner (SHP), fibroblast growth factors receptor 4 (FGFR4) and hepatocyte nuclear factor 4- $\alpha$ (HNF4 $\alpha)$. As results showed, LXR $\alpha$ and HNF4 $\alpha$ did not change in each group (data not show). However, FGFR4 increased while FXR and SHP decreased in both $o b / o b$ mice and ApoC3 transgenic mice, which changes were reversed by GLPP (Fig. 4 e-h). Since nuclear receptors mediated regulation of CYP7A1 have been shown to influence several aspects of lipid

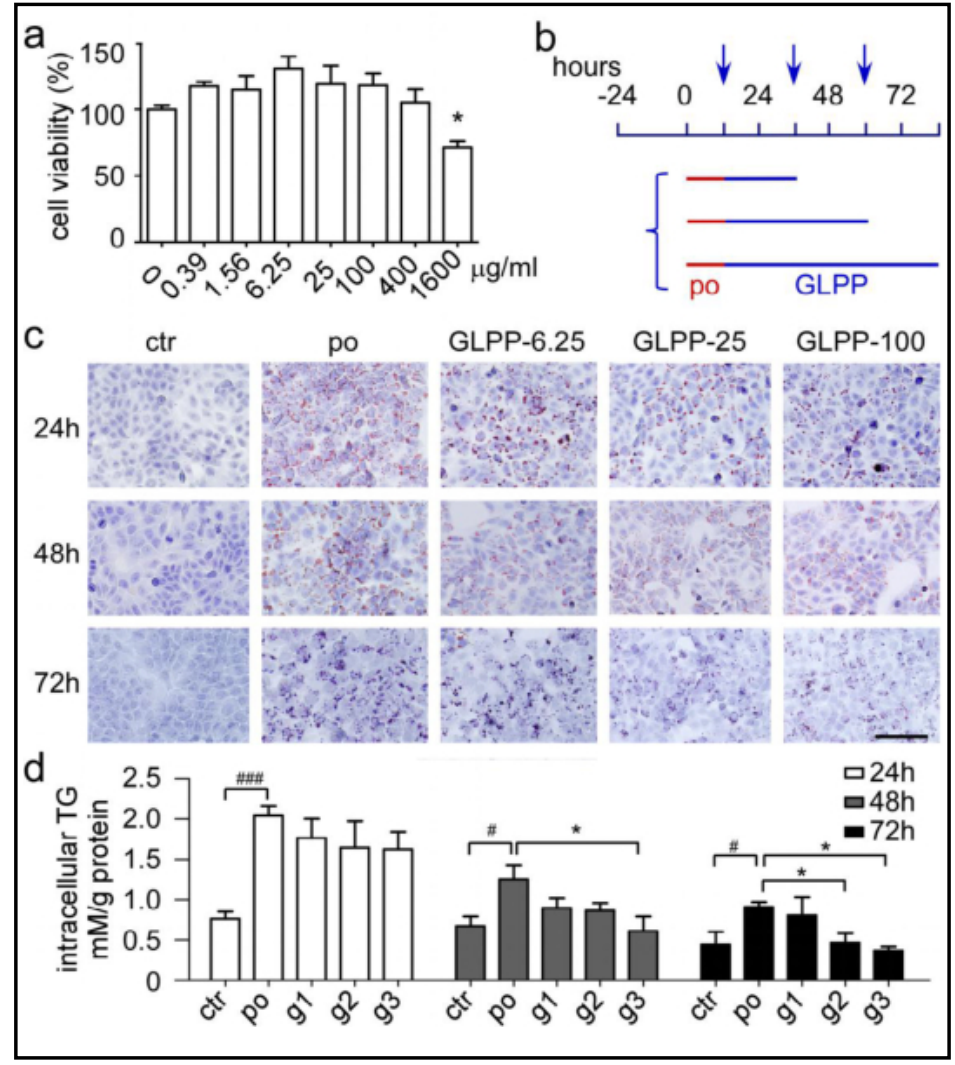

Fig. 5. GLPP reduces intracellular lipid in HepG2 cells induced by palmitic acid and oleic acid. (a) Cytotoxicity of GLPP on HepG2 cells $(n=5)$. (b) A schematic diagram showing the procedure of treatment. (c) Oil red 0 staining of cells. Scale bar, $200 \mu \mathrm{m}$. (d) Intracellular TG content $(\mathrm{n}=6)$. Quantitative data are presented as mean \pm SEM. ${ }^{*} \mathrm{P}<0.05$, ${ }^{\#} \mathrm{P}<0.05$, ${ }^{\# \#} \mathrm{P}<0.01$, ${ }^{\# \#} \mathrm{P}<0.001$. 
metabolism, we then analyzed the expression of sterol regulatory element binding protein 1c (SREBP-1c), fatty acid synthase (FAS) and acetyl-CoA carboxylase (ACC), and found that they both increased in $o b / o b$ mice and ApoC3 transgenic mice while GLPP reversed their expression levels (Fig. 4 e-h). Lipid accumulation is not merely determined by lipogenesis, but also lies on lipid oxidation. Carnitine palmitoyltransferase 1A (CPT1A), mediating fatty acid oxidation, was tested but with no statistical difference between all groups (data not show). These data suggest that GLPP may regulate BAs synthesis dependent on FXR-SHP/ FGF and inhibit the downstream pathway of lipogenesis.

\section{GLPP reduces lipid accumulation and intracellular TG levels in HepG2 cells}

Next, we studied the effect of GLPP on NAFLD in vitro. GLPP was nontoxic under 1600 $\mu \mathrm{g} / \mathrm{ml}$. GLPP at concentrations of $6.25 \mu \mathrm{g} / \mathrm{ml}, 25 \mu \mathrm{g} / \mathrm{ml}$ and $100 \mu \mathrm{g} / \mathrm{ml}$ were used for further experiment (Fig. 5 a). HepG2 cells induced by palmitic acid and oleic acid, an in vitro hepatosteatosis model were used. After $12 \mathrm{~h}$ of palmitic acid and oleic acid exposure, HepG2 cells were treated with GLPP for $24 \mathrm{~h}, 48 \mathrm{~h}$ or $72 \mathrm{~h}$ (Fig. $5 \mathrm{~b}$ ). As results showed, palmitic acid and oleic acid significantly increased the accumulation of lipid droplets and the content of intracellular TG in HepG2 cells, while GLPP reduced them in a dose and time-dependent manner. (Fig. 5 c, d).

\section{GLPP reduces accumulated lipid accumulation and intracellular TG levels in primary} hepatocytes

To confirm that GLPP could protect against steatosis, primary hepatocytes isolated from C57 mice were induced with palmitic acid and oleic acid. After exposure to palmitic acid and oleic acid for $12 \mathrm{~h}$, primary hepatocytes were further cultured with GLPP for $24 \mathrm{~h}$ or 48 h (Fig. 6 a). As oil red 0 staining and intracellular TG detection showed, GLPP significantly reduced the number of lipid droplets and the level of intracellular TG, which suggests that GLPP alleviates liver steatosis in primary hepatocytes (Fig. 6 b, c).

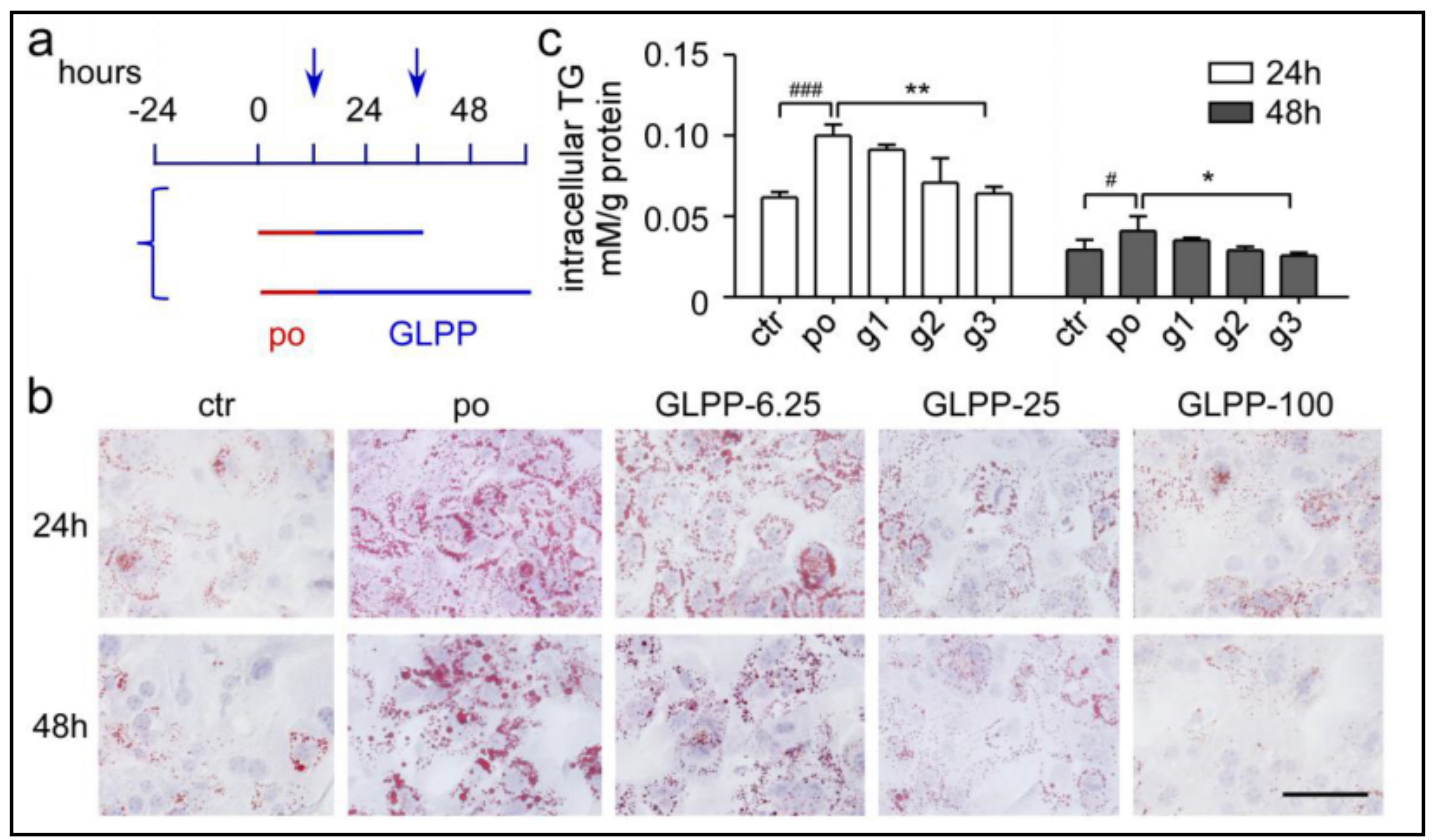

Fig. 6. GLPP reduces intracellular lipid levels in primarily cultured hepatocytes induced by palmitic acid and oleic acid. (a) A schematic diagram showing the procedure of the treatment. (b) Oil red 0 staining of cells. Scale bar, $200 \mu \mathrm{m}$. (c) Intracellular TG content $(\mathrm{n}=6)$. Quantitative data are presented as mean \pm SEM.

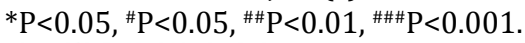




\section{Cellular Physiology Cell Physiol Biochem 2018;49:1204-1220

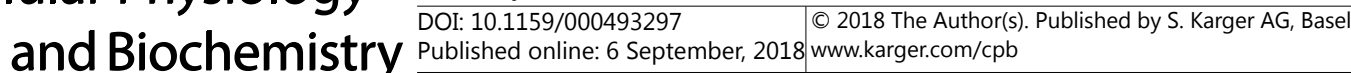 \\ Zhong et al.: GLPP Treats Non-Alcohol Fatty Liver Disease}

\section{Discussion}

Using $o b / o b$ mice and ApoC3 transgenic mouse models, we found that GLPP alleviated hepatic steatosis, liver dysfunction, dyslipidemia and liver IR induced by obesity and hypertriglyceridemia. According to metabolomics, KEGG and PIUmet analysis, GLPP mainly regulated the classic pathway of BAs synthesis in a FXR-SHP/FGF dependent mechanism to protect against NAFLD. In vitro, hepatosteatosis models of HepG2 cells and primary hepatocytes induced by palmitic acid and oleic acid confirmed the therapeutic effect of GLPP against NAFLD.

$O b / o b$ mice develop spontaneous hepatic steatosis, showing hyperglycemia, IR and hyperinsulinemia, which are widely used to study NAFLD [21]. NAFLD and IR have recently been found to be associated with increased plasma concentrations of APOC3 in humans carrying single nucleotide polymorphisms within the insulin response element of the APOC3 gene [22]. APOC3-transgenic mice are hypertriglyceridemic, with marked elevation of triglycerides, cholesterols, and nonesterified fatty acids in plasma and mild steatosis, which are common models for studying NAFLD [23]. Additionally, HepG2 cell and primary hepatocyte induced with palmitic acid and oleic acid are widely used in the study of NAFLD and related metabolic disorders [24-26]. Here, we chose these two animal models and two cell hepatosteatosis models to study the protective effect of GLPP against the benign stage of NAFLD excluding disturbance of dietary and risk factors inducing NASH.

The earliest stage of NAFLD is hepatic steatosis, which is characterized by the deposition of TG as lipid droplets in the cytoplasm of hepatocytes. Lipid accumulation is a hallmark of NAFLD. Lowering lipid is accepted as an effective measure to ameliorate NAFLD [27-29]. Previous study showed that a proteoglycan named Fudan-Yueyang-Ganoderma lucidum (FYGL) extracted from Ganoderma lucidum had antihyperlipidemic effect, but the author paid most attention to the effect of FYGL on antidiabetic while no further study on liver protection [30]. Accumulated studies focused on the immuno-modulating or anti-oxidant effect of ganoderma lucidum on liver injury induced by hepatotoxic drugs or ethanol-induced acute liver injury [31,32], but little attention focused on NAFLD. We made a deep research on the protection of GLPP against NAFLD, which is the main risk factor of chronic liver diseases. In both $o b / o b$ mice and ApoC3 transgenic mice, GLPP significantly modified dyslipidemia, reduced the content of TG and TC in the liver and alleviated steatosis. Also, in HepG2 and primary hepatocytes, the numbers of lipid droplets and levels of intracellular TG reduced by GLPP in a dose- and time-dependent manner. The results suggest that GLPP has an effect in modifying dyslipidemia and treating steatosis.

Simple hepatic steatosis reflects the accumulation of TG in the liver, as result of influx of lipids and de novo lipogenesis exceeding the export of lipids in the forms of lipoproteins. IR is considered as a primary driving force for hepatic steatosis by promoting lipolysis of peripheral adipose tissue and increasing hepatic uptake of free fatty acids for de novo lipogenesis [33]. Hepatic steatosis and IR are mutually promoted pathological events in metabolic disorders [34]. IR plays a pivotal role in the pathogenic switch of fatty liver and interventions that ameliorate IR lead to lower insulin levels and decreased liver fat content. Many studies support a direct causal relationship between IR and hepatic steatosis [35]. Previous study showed that extracts of ganoderma lucidum possessed antidiabetic activity via down-regulating hepatic glucose regulatory enzymes in diabetic mice [36]. In our study, p-IRS2 and p-AKT2 decreased in the liver of both ob/ob mice and ApoC3 transgenic mice, while p-GSK3 $\beta$ increased, which suggests that liver has undergone IR. GLPP alleviated the liver IR by increasing the phosphorylation of IRS2 and AKT2 while inhibiting the activation of GSK3 3 .

NAFLD is a complex metabolic disease accompanied by changes of a series of metabolites. Profiling of metabolic products (metabolic phenotyping or metabotyping) has provided new insights into metabolic syndrome and NAFLD. In the present study, we prepared serum samples of $o b / o b$ mice for metabolite profiling analysis to help us analyze and interpret metabolic signatures in terms of metabolic pathways and protein interaction 
networks and to identify the genomic and metagenomic determinants of metabolism. Metabolomics analysis revealed that pathways of glycerophospholipid metabolism, taurine and hypotaurine metabolism, fatty acid metabolism and primary bile acid biosynthesis possibly participated in the protection of GLPP against NAFLD. We then employed a networkbased approach PIUMet, which encloses a sub-metabolic network defined by upregulated metabolites or down-regulated metabolites. Key enzymes were enriched and classified into pathways of glycerophospholipid metabolism, TG synthesis, cholesterol metabolism and BAs metabolism. Further confirmation revealed that CYP7A1 and CYP8B1 significantly decreased in $o b / o b$ mice and ApoC3 mice while GLPP reversed their expression levels. However, LPGAT1, LCAT, CLC and CYP7B1 had no significant difference. The results indicate that GLPP mainly regulates the classic pathway of BAs synthesis. Extensive researches had unveiled new functions of BAs not only acted as signaling molecules, but also participated in a variety of pathways that regulate BA, glucose, and lipid homeostasis [37]. CYP7A1 is the rate-limiting enzyme of BAs synthesis and is regulated by a number of nuclear receptors, such as FXR, LXR and SHP, which play vital roles in NAFLD [38-40]. FXR is a master regulator maintaining the homeostasis of BAs. In response to elevated levels of intracellular BAs, FXR is activated to induce protective gene expression circuits against BAs toxicity in the liver and intestine [41]. FXR induces SHP in the liver, and fibroblast growth factors 15/19 (FGF15/19) that binds with liver FGFR4 via an endocrine mode after its secretion from the intestine. Agonists or antagonists of nuclear receptors serves as therapeutic agents for reversing or halting liver steatosis and its related disorders $[42,43]$. Both signal inhibits CYP7A1, the ratecontrolling enzyme in the de novo synthesis of BAs [44, 45]. As our results showed, compared to control mice, FGFR4 was up-regulated while FXR and SHP were down-regulated in $o b /$ $o b$ mice and ApoC3 transgenic mice while GLPP reversed their expression levels. However, LXR and HNF $4 \alpha$ remained unchanged. These results suggest that the protection of GLPP on NAFLD may attribute to the regulation of BAs dependent on FXR-SHP/FGF.

Extended studies had reported that FXR and FGF participated in lipid metabolism [46]. Previous studies showed that plasma TG and cholesterol levels were elevated in FXR-null mice [47]. In addition, FXR had been shown to regulate a set of genes involved in lipoprotein metabolism such as SREBP1, suggesting that FXR played a key role in regulating hepatic lipid metabolism [48]. In addition, FGF participated in inter-organ endocrine signaling axes that were critical for maintaining whole-body homeostasis, as they govern BAs, glucose and lipid metabolism $[49,50]$. Also, a previous study revealed that FGF19 was a novel factor that inhibited hepatic fatty acid synthesis [51]. SREBP-1c, a key transcription factor regulating hepatic lipid metabolism preferentially enhances the expression of genes involved in fatty acid synthesis, had been proposed to have great potential for NAFLD treatment [52]. Previous study had shown that SREBP-1c made to TG accumulation in insulin-resistant livers of $o b /$ $o b$ mice [53]. SREBP-1c expression leads to the transcriptional activation of all lipogenic genes such as FAS and ACC. Activation of ACC converts acetyl-CoA to malonyl-CoA, which is used by FAS to form palmitic acid. Palmitic acid transforms to different fatty acids, which are used to synthesize triacylglycerol. In our study, the expression of SREBP-1c, FAS and ACC, key enzymes mediating fatty acid synthesis, were increased in $o b / o b$ mice and ApoC3 transgenic mice while GLPP decreased their expression. Data suggest that GLPP significantly inhibits the lipogenesis in the liver.

Hepatic TG levels are not only determined by the fatty acid synthesis rate via SREBP$1 c$, but also fatty acid $\beta$-oxidation $[54,55]$. The enzyme CPT1A (expressed in the liver), controlling mitochondrial $\beta$-oxidation, participates in the mechanism of protection on steatosis [56]. Our data showed no statistic changes of CPT1A in both ob/ob mice and ApoC3 transgenic mice, which suggested that the protection of GLPP on NAFLD is mainly attributed to the inhibition of lipogenesis.

The present study suggests a hypothesis as followed. Under condition of steatosis, the expression of CYP7A1 and CYP8B1 are down-regulated, which reduces BAs synthesis. BAs are endogenous ligand of FXR and bind to it, which induces the expression of SHP. Decreased synthesis of BAs leads to less activation of FXR and thus reduces SHP. SHP regulates

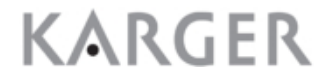




\section{Cellular Physiology Cell Physiol Biochem 2018;49:1204-1220 and Biochemistry $\begin{aligned} & \text { DOI: 10.1159/000493297 } \\ & \text { Published online: 6 September, } 2018 \text { wwwwkarger.com/cpb }\end{aligned}$ \\ Zhong et al.: GLPP Treats Non-Alcohol Fatty Liver Disease}

SREBP1c negatively. Decreased SHP thus facilitates lipogenesis by up-regulating genes of SREBP-1c, FAS, ACC and aggravates NAFLD. Besides, FGF-15 is a secreted growth factor that signals through the FGFR4 cell-surface receptor tyrosine kinase [57]. Secreted FGF15 from enterocytes travels to the liver via portal vein and binds to FGFR4 in the hepatocytes to activate JNK signaling pathway, which in turn suppresses CYP7A1 expression and further reduces BAs synthesis. Furthermore, liver insulin insensitivity results in low expression of IRS2, p-IRS2 and reduces activity of AKT2. GSK3 $\beta$ is a critical downstream element of AKT. Increased phosphorylation of GSK3 $\beta$ inactivates glycogen synthase (GS) and destroys glucose hemostasis, which finally aggravates NAFLD. GLPP reverses the expression changes of these proteins involved in BAs synthesis, lipogenesis and IR, which ultimately alleviates NAFLD.

\section{Conclusion}

In conclusion, the present study firstly identified a therapeutic role of GLPP on NAFLD via regulating bile acids synthesis dependent on FXR-SHP/FGF pathway and inhibiting its downstream pathway of fatty acid synthesis, which suggests that GLPP can be a candidate drug for treating NAFLD.

\section{Acknowledgements}

DZ and BY designed the experiments. DZ, BH, GW and SZ performed the research. DZ and ZX analyzed the data. DZ, ZX, HZ and BY interpreted the results. DZ, ZX and BY prepared figures and drafted the manuscript. DZ, ZX, BH, SZ and BY edited the manuscript. All authors read and approved the final manuscript.

This work was supported by National Natural Science Foundation of China grants 81330074, 81620108029 and 81261160507, and Beijing Natural Science Foundation grant 7172113.

\section{Disclosure Statement}

The authors declare that they have no competing interests.

\section{References}

1 Weiss J, Rau M, Geier A: Non-alcoholic fatty liver disease: Epidemiology, clinical course, investigation, and treatment. Dtsch Arztebl Int 2014;111:447-452.

-2 Pappachan JM, Antonio FA, Edavalath M, Mukherjee A: Non-alcoholic fatty liver disease: A diabetologist's perspective. Endocrine 2014;45:344-353.

-3 Fazel Y, Koenig AB, Sayiner M, Goodman ZD, Younossi ZM: Epidemiology and natural history of nonalcoholic fatty liver disease. Metabolism 2016;65:1017-1025.

4 Musso G, Gambino R, Cassader M: Non-alcoholic fatty liver disease from pathogenesis to management: An update. Obes Rev 2010;11:430-445.

-5 Klupp NL, Chang D, Hawke F, Kiat H, Cao H, Grant SJ, Bensoussan A: Ganoderma lucidum mushroom for the treatment of cardiovascular risk factors. Cochrane Database Syst Rev 2015:D7259.

6 Tsao SM, Hsu HY: Fucose-containing fraction of Ling-Zhi enhances lipid rafts-dependent ubiquitination of TGFbeta receptor degradation and attenuates breast cancer tumorigenesis. Sci Rep 2016;6:36563.

7 Amen YM, Zhu Q, Tran HB, Afifi MS, Halim AF, Ashour A, Mira A, Shimizu K: Lucidumol C, a new cytotoxic lanostanoid triterpene from Ganoderma lingzhi against human cancer cells. J Nat Med 2016;70:661-666. 


\section{Cellular Physiology Cell Physiol Biochem 2018;49:1204-1220 \begin{tabular}{l|l|l} 
and Biochemistry Published online: 6 September, 2018 & $\begin{array}{l}\text { (c) } 2018 \text { The Author(s). Published by S. Karger AG, Basel } \\
\text { ww.karger.com/cpb }\end{array}$
\end{tabular} \\ Zhong et al.: GLPP Treats Non-Alcohol Fatty Liver Disease}

8 Li A, Shuai X, Jia Z, Li H, Liang X, Su D, Guo W: Ganoderma lucidum polysaccharide extract inhibits hepatocellular carcinoma growth by downregulating regulatory $\mathrm{T}$ cells accumulation and function by inducing microRNA-125b. J Transl Med 2015;13:100.

-9 Wu H, Tang S, Huang Z, Zhou Q, Zhang P, Chen Z: Hepatoprotective Effects and Mechanisms of Action of Triterpenoids from Lingzhi or Reishi Medicinal Mushroom Ganoderma lucidum (Agaricomycetes) on alpha-Amanitin-Induced Liver Injury in Mice. Int J Med Mushrooms 2016;18:841-850.

10 Zhong D, Wang H, Liu M, Li X, Huang M, Zhou H, Lin S, Lin Z, Yang B: Ganoderma lucidum polysaccharide peptide prevents renal ischemia reperfusion injury via counteracting oxidative stress. Sci Rep 2015;5:16910.

11 Ma HT, Hsieh JF, Chen ST: Anti-diabetic effects of Ganoderma lucidum. Phytochemistry 2015;114:109-113.

-12 Chang CJ, Lin CS, Lu CC, Martel J, Ko YF, Ojcius DM, Tseng SF, Wu TR, Chen YY, Young JD, Lai HC: Ganoderma lucidum reduces obesity in mice by modulating the composition of the gut microbiota. Nat Commun 2015;6:7489.

13 Tran HB, Yamamoto A, Matsumoto S, Ito H, Igami K, Miyazaki T, Kondo R, Shimizu K: Hypotensive effects and angiotensin-converting enzyme inhibitory peptides of reishi (Ganoderma lingzhi) auto-digested extract. Molecules 2014;19:13473-13485.

14 Cao QZ, Lin ZB: Antitumor and anti-angiogenic activity of Ganoderma lucidum polysaccharides peptide. Acta Pharmacol Sin 2004;25:833-838.

15 Cao QZ, Lin ZB: Ganoderma lucidum polysaccharides peptide inhibits the growth of vascular endothelial cell and the induction of VEGF in human lung cancer cell. Life Sci 2006;78:1457-1463.

-16 Chiu HF, Fu HY, Lu YY, Han YC, Shen YC, Venkatakrishnan K, Golovinskaia O, Wang CK: Triterpenoids and polysaccharide peptides-enriched Ganoderma lucidum: A randomized, double-blind placebo-controlled crossover study of its antioxidation and hepatoprotective efficacy in healthy volunteers. Pharm Biol 2017;55:1041-1046.

17 Pirhaji L, Milani P, Leidl M, Curran T, Avila-Pacheco J, Clish CB, White FM, Saghatelian A, Fraenkel E: Revealing disease-associated pathways by network integration of untargeted metabolomics. Nat Methods 2016;13:770-776.

18 Wang Q, Jiang L, Wang J, Li S, Yu Y, You J, Zeng R, Gao X, Rui L, Li W, Liu Y: Abrogation of hepatic ATPcitrate lyase protects against fatty liver and ameliorates hyperglycemia in leptin receptor-deficient mice. Hepatology 2009;49:1166-1175.

19 Donnelly KL, Smith CI, Schwarzenberg SJ, Jessurun J, Boldt MD, Parks EJ: Sources of fatty acids stored in liver and secreted via lipoproteins in patients with nonalcoholic fatty liver disease. J Clin Invest 2005;115:1343-1351.

20 Pollak M: The insulin and insulin-like growth factor receptor family in neoplasia: An update. Nat Rev Cancer 2012;12:159-169.

-21 Bray GA, York DA: Hypothalamic and genetic obesity in experimental animals: An autonomic and endocrine hypothesis. Physiol Rev 1979;59:719-809.

22 Duseja A, Aggarwal R: APOC3 and PNPLA3 in non-alcoholic fatty liver disease: Need to clear the air. J Gastroenterol Hepatol 2012;27:848-851.

-23 Lee HY, Birkenfeld AL, Jornayvaz FR, Jurczak MJ, Kanda S, Popov V, Frederick DW, Zhang D, Guigni B, Bharadwaj KG, Choi CS, Goldberg IJ, Park JH, Petersen KF, Samuel VT, Shulman GI: Apolipoprotein CIII overexpressing mice are predisposed to diet-induced hepatic steatosis and hepatic insulin resistance. Hepatology 2011;54:1650-1660.

-24 Zeng L, Tang W, Yin J, Feng L, Li Y, Yao X, Zhou B: Alisol a 24-Acetate prevents hepatic steatosis and metabolic disorders in HepG2 cells. Cell Physiol Biochem 2016;40:453-464.

-25 Hetherington AM, Sawyez CG, Zilberman E, Stoianov AM, Robson DL, Borradaile NM: Differential lipotoxic effects of palmitate and oleate in activated human hepatic stellate cells and epithelial hepatoma cells. Cell Physiol Biochem 2016;39:1648-1662.

26 Al FF, Fayyaz S, Japtok L, Kleuser B: Involvement of sphingosine 1-Phosphate in Palmitate-Induced NonAlcoholic fatty liver disease. Cell Physiol Biochem 2016;40:1637-1645.

-27 Schweiger M, Romauch M, Schreiber R, Grabner GF, Hutter S, Kotzbeck P, Benedikt P, Eichmann TO, Yamada S, Knittelfelder O, Diwoky C, Doler C, Mayer N, De Cecco W, Breinbauer R, Zimmermann R, Zechner R: Pharmacological inhibition of adipose triglyceride lipase corrects high-fat diet-induced insulin resistance and hepatosteatosis in mice. Nat Commun 2017;8:14859. 


\section{Cellular Physiology Cell Physiol Biochem 2018;49:1204-1220

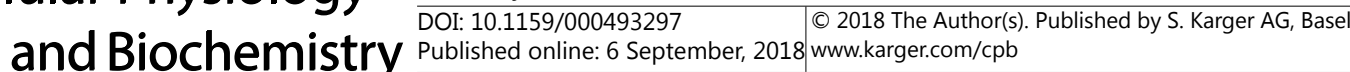 \\ Zhong et al.: GLPP Treats Non-Alcohol Fatty Liver Disease}

28 Wu YL, Zhu YB, Huang RD, Peng XE, Lin X: Multiple MicroRNAs ameliorate hepatocyte steatosis and injury by suppressing FABP1 expression. Cell Physiol Biochem 2017;44:2243-2255.

29 Wu GY, Rui C, Chen JQ, Sho E, Zhan SS, Yuan XW, Ding YT: MicroRNA-122 Inhibits Lipid Droplet Formation and Hepatic Triglyceride Accumulation via Yin Yang 1. Cell Physiol Biochem 2017;44:1651-1664.

-30 Wang CD, Teng BS, He YM, Wu JS, Pan D, Pan LF, Zhang D, Fan ZH, Yang HJ, Zhou P: Effect of a novel proteoglycan PTP1B inhibitor from Ganoderma lucidum on the amelioration of hyperglycaemia and dyslipidaemia in db/db mice. Br J Nutr 2012;108:2014-2025.

-31 Liu YJ, Du JL, Cao LP, Jia R, Shen YJ, Zhao CY, Xu P, Yin GJ: Anti-inflammatory and hepatoprotective effects of Ganoderma lucidum polysaccharides on carbon tetrachloride-induced hepatocyte damage in common carp (Cyprinus carpio L.). Int Immunopharmacol 2015;25:112-120.

-32 Jang SH, Cho SW, Yoon HM, Jang KJ, Song CH, Kim CH: Hepatoprotective Evaluation of Ganoderma lucidum Pharmacopuncture: In vivo Studies of Ethanol-induced Acute Liver Injury. J Pharmacopuncture 2014;17:16-24.

33 Browning JD, Horton JD: Molecular mediators of hepatic steatosis and liver injury. J Clin Invest 2004;114:147-152.

34 Samuel VT, Shulman GI: Mechanisms for insulin resistance: Common threads and missing links. Cell 2012;148:852-871.

35 Hebbard L, George J: Animal models of nonalcoholic fatty liver disease. Nat Rev Gastroenterol Hepatol 2011;8:35-44.

-36 Xiao C, Wu Q, Zhang J, Xie Y, Cai W, Tan J: Antidiabetic activity of Ganoderma lucidum polysaccharides F31 down-regulated hepatic glucose regulatory enzymes in diabetic mice. J Ethnopharmacol 2017;196:47-57.

37 Watanabe M, Houten SM, Mataki C, Christoffolete MA, Kim BW, Sato H, Messaddeq N, Harney JW, Ezaki O, Kodama T, Schoonjans K, Bianco AC, Auwerx J: Bile acids induce energy expenditure by promoting intracellular thyroid hormone activation. Nature 2006;439:484-489.

-38 Scotti E, Gilardi F, Godio C, Gers E, Krneta J, Mitro N, De Fabiani E, Caruso D, Crestani M: Bile acids and their signaling pathways: Eclectic regulators of diverse cellular functions. Cell Mol Life Sci 2007;64:2477-2491.

-39 Zhou H, Hylemon PB: Bile acids are nutrient signaling hormones. Steroids 2014;86:62-68.

40 Wang L, Zhang N, Wang Z, Ai DM, Cao ZY, Pan HP: Decreased MiR-155 level in the peripheral blood of NonAlcoholic fatty liver disease patients may serve as a biomarker and may influence LXR activity. Cell Physiol Biochem 2016;39:2239-2248.

41 Sinal CJ, Tohkin M, Miyata M, Ward JM, Lambert G, Gonzalez FJ: Targeted disruption of the nuclear receptor FXR/BAR impairs bile acid and lipid homeostasis. Cell 2000;102:731-744.

42 Jiang C, Xie C, Lv Y, Li J, Krausz KW, Shi J, Brocker CN, Desai D, Amin SG, Bisson WH, Liu Y, Gavrilova O, Patterson AD, Gonzalez FJ: Intestine-selective farnesoid X receptor inhibition improves obesity-related metabolic dysfunction. Nat Commun 2015;6:10166.

-43 Xu Y, Zalzala M, Xu J, Li Y, Yin L, Zhang Y: A metabolic stress-inducible miR-34a-HNF4alpha pathway regulates lipid and lipoprotein metabolism. Nat Commun 2015;6:7466.

44 Modica S, Petruzzelli M, Bellafante E, Murzilli S, Salvatore L, Celli N, Di Tullio G, Palasciano G, Moustafa T, Halilbasic E, Trauner M, Moschetta A: Selective activation of nuclear bile acid receptor FXR in the intestine protects mice against cholestasis. Gastroenterology 2012;142:355-365.

45 Inagaki T, Choi M, Moschetta A, Peng L, Cummins CL, McDonald JG, Luo G, Jones SA, Goodwin B, Richardson JA, Gerard RD, Repa JJ, Mangelsdorf DJ, Kliewer SA: Fibroblast growth factor 15 functions as an enterohepatic signal to regulate bile acid homeostasis. Cell Metab 2005;2:217-225.

46 Abd EA, Ramadan BK, Schaalan MF, Tolba AM: A novel role of SIRT1/ FGF-21 in taurine protection against cafeteria Diet-Induced steatohepatitis in rats. Cell Physiol Biochem 2017;43:644-659.

47 Zhang Y, Lee FY, Barrera G, Lee H, Vales C, Gonzalez FJ, Willson TM, Edwards PA: Activation of the nuclear receptor FXR improves hyperglycemia and hyperlipidemia in diabetic mice. Proc Natl Acad Sci U S A 2006;103:1006-1011.

48 Lambert G, Amar MJ, Guo G, Brewer HJ, Gonzalez FJ, Sinal CJ: The farnesoid X-receptor is an essential regulator of cholesterol homeostasis. J Biol Chem 2003;278:2563-2570.

-49 Potthoff MJ, Boney-Montoya J, Choi M, He T, Sunny NE, Satapati S, Suino-Powell K, Xu HE, Gerard RD, Finck BN, Burgess SC, Mangelsdorf DJ, Kliewer SA: FGF15/19 regulates hepatic glucose metabolism by inhibiting the CREB-PGC-1alpha pathway. Cell Metab 2011;13:729-738. 


\section{Cellular Physiology Cell Physiol Biochem 2018;49:1204-1220

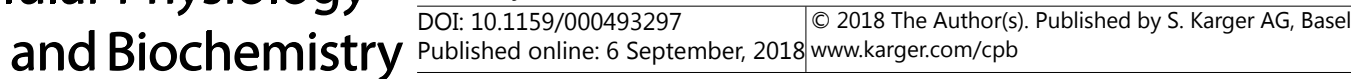 \\ Zhong et al.: GLPP Treats Non-Alcohol Fatty Liver Disease}

50 Xu J, Lloyd DJ, Hale C, Stanislaus S, Chen M, Sivits G, Vonderfecht S, Hecht R, Li YS, Lindberg RA, Chen JL, Jung DY, Zhang Z, Ko HJ, Kim JK, Veniant MM: Fibroblast growth factor 21 reverses hepatic steatosis, increases energy expenditure, and improves insulin sensitivity in diet-induced obese mice. Diabetes 2009;58:250-259.

51 Bhatnagar S, Damron HA, Hillgartner FB: Fibroblast growth factor-19, a novel factor that inhibits hepatic fatty acid synthesis. J Biol Chem 2009;284:10023-10033.

-52 Ahmed MH, Byrne CD: Modulation of sterol regulatory element binding proteins (SREBPs) as potential treatments for non-alcoholic fatty liver disease (NAFLD). Drug Discov Today 2007;12:740-747.

53 Shimomura I, Bashmakov Y, Horton JD: Increased levels of nuclear SREBP-1c associated with fatty livers in two mouse models of diabetes mellitus. J Biol Chem 1999;274:30028-30032.

54 Horton JD, Goldstein JL, Brown MS: SREBPs: Activators of the complete program of cholesterol and fatty acid synthesis in the liver. J Clin Invest 2002;109:1125-1131.

55 Shao M, Shan B, Liu Y, Deng Y, Yan C, Wu Y, Mao T, Qiu Y, Zhou Y, Jiang S, Jia W, Li J, Li J, Rui L, Yang L, Liu Y: Hepatic IRE1alpha regulates fasting-induced metabolic adaptive programs through the XBP1s-PPARalpha axis signalling. Nat Commun 2014;5:3528.

56 Derdak Z, Villegas KA, Harb R, Wu AM, Sousa A, Wands JR: Inhibition of p53 attenuates steatosis and liver injury in a mouse model of non-alcoholic fatty liver disease. J Hepatol 2013;58:785-791.

57 Holt JA, Luo G, Billin AN, Bisi J, McNeill YY, Kozarsky KF, Donahee M, Wang DY, Mansfield TA, Kliewer SA, Goodwin B, Jones SA: Definition of a novel growth factor-dependent signal cascade for the suppression of bile acid biosynthesis. Genes Dev 2003;17:1581-1591. 